\title{
Infrared spectral classification of normal stars ${ }^{\star}$
}

\author{
A. M. Heras ${ }^{1}$, R. F. Shipman ${ }^{2}$, S. D. Price ${ }^{3}$, Th. de Graauw ${ }^{2}$, H. J. Walker ${ }^{4}$, M. Jourdain de Muizon ${ }^{\star \star, 5,6}$, \\ M. F. Kessler ${ }^{7}$, T. Prusti ${ }^{1}$, L. Decin ${ }^{\star \star \star, 8}$, B. Vandenbussche ${ }^{8}$, and L. B. F. M. Waters ${ }^{9}$ \\ 1 Astrophysics Missions Division, Research and Scientific Support Department of ESA, ESTEC, PO Box 299, \\ 2200 AG Noordwijk, The Netherlands \\ e-mail: aheras@rssd.esa.int \\ 2 Space Research Organization of The Netherlands, PO Box 800, 9700 AV Groningen, The Netherlands \\ 3 Air Force Research Laboratory/Space Vehicles Directorate, AFRL/VSB, Hanscom AFB, USA \\ 4 Space Science Department, Rutherford Appleton Laboratory, Chilton, Oxon OX11 0QX, UK \\ 5 LESIA, Observatoire de Paris, 92190 Meudon, France \\ ${ }^{6}$ Laboratorio de Astrofísica Espacial y Física Fundamental, INTA, PO Box 50727, 28080 Madrid, Spain \\ 7 Science Operations and Data Systems Division, Research and Scientific Support Department of ESA, ESTEC, PO Box 299, \\ 2200 AG Noordwijk, The Netherlands \\ ${ }^{8}$ Instituut voor Sterrenkunde, K.U. Leuven, Celestijnenlaan 200B, 3001 Leuven, Belgium \\ 9 Astronomical Institute "Anton Pannekoek", University of Amsterdam, Kruislaan 403, 1098 SJ Amsterdam, The Netherlands
}

Received 7 May 2002 / Accepted 1 August 2002

\begin{abstract}
Moderate resolution $(\approx 400) 2.38-45.2 \mu \mathrm{m}$ infrared spectra of stars without dust features were obtained with the Short Wavelength Spectrometer (SWS) on board the Infrared Space Observatory (ISO). The observations are part of a larger program with the objective to extend and refine the current infrared classification schemes. In particular, our data provide the basis for a more detailed classification of the 1.N-1.NO sources (ordinary and oxygen rich naked stars) as defined by Kraemer et al. (2002) in a comprehensive classification of the ISO-SWS spectra. For our analysis, the continuum was determined by fitting Engelke's function (Engelke 1992) to the SWS data. The stellar angular diameters derived from these estimates of the continuum are in good agreement with values obtained by other methods. Analysis of the equivalent widths of the CO fundamental and first overtone molecular bands, the $\mathrm{SiO}$ fundamental and first overtone, as well as the $\mathrm{H}_{2} \mathrm{O}$ bending mode band as a function of MK class, reveals that there is sufficient information in the SWS spectra to distinguish between hot (B, A, F) and cool stars. Furthermore, it is possible to determine the spectral type for the $\mathrm{G}, \mathrm{K}$ and $\mathrm{M}$ giants, and subtype ranges in a sequence of $\mathrm{K}$ and $\mathrm{M}$ giants. The equivalent widths of the $\mathrm{CO}$ and $\mathrm{SiO}$ bands are found to be well correlated in $\mathrm{K}$ and $\mathrm{M}$ stars, such that the equivalent widths of the $\mathrm{CO}$ fundamental, the $\mathrm{SiO}$ first overtone and the $\mathrm{SiO}$ fundamental can be reasonably well extrapolated from the depth of the CO first overtone. We have identified two stars, HR 365 and V Nor, whose mid-infrared spectrum does not correspond to their respective optical classification. HR 365 may have a late M companion, which dominates the observed infrared spectrum while V Nor is a late type giant that was included because its spectrum was classified as featureless under the IRAS LRS scheme. According to Kraemer et al. (2002), V Nor has a thin dust shell, which distorts the analysis of its mid-infrared absorption bands.
\end{abstract}

Key words. stars: atmospheres - stars: evolution - stars: fundamental parameters - infrared: stars

Send offprint requests to: A. M. Heras,

e-mail: aheras@rssd.esa.int

* Based on observations with ISO, an ESA project with instruments funded by ESA Member States (especially the PI countries: France, Germany, The Netherlands and the UK) and with the participation of ISAS and NASA.

$\star \star$ now at Leiden Observatory, PO Box 9513, 2300 RA Leiden, The Netherlands.

$\star \star \star$ Postdoctoral Fellow of the Fund for Scientific Research, Flanders.

\section{Introduction}

The characterization and analysis of stellar infrared spectra is an essential tool in understanding the physical and chemical processes taking place in stellar atmospheres, especially for late-type stars. Detailed studies have been carried out in the near-infrared region of the spectrum that have correlated the observed spectral features with the MK class and defined spectral indices for a classification in the infrared (e.g. Smith \& Lambert 1985; Origlia et al. 1993; Andrillat et al. 1995; 
Morris et al. 1996; Wallace \& Hinkle 1996; Meyer et al. 1998). The IRAS satellite obtained low resolution (20 to 60) 7.7$22.6 \mu \mathrm{m}$ mid-infrared spectra of the brighter point sources in the sky with the Low-Resolution Spectrometer (LRS). These spectra were used to create the LRS classification scheme (IRAS Science Team 1986). Further classification work has been done on specific types of LRS sources. For example, Sloan \& Price (1998) presented the classification of a LRS sample of oxygen-rich variables, and showed the existence of a silicate dust sequence in their dust shells. More recently, the Short Wavelength Spectrometer (SWS) on the Infrared Space Observatory (ISO) provided observations that covered a much broader wavelength range $(2.38-45.2 \mu \mathrm{m})$ at higher spectral resolution and sensitivity than the LRS. The results in this paper are part of a more extensive program that attempts to extend and improve the LRS classification by analyzing the ISO-SWS full range spectra obtained during the ISO mission (Kraemer et al. 2002). A parallel program (Vandenbussche et al. 1999, 2002) for the classification of sources based on near-infrared $(2.36-4.1 \mu \mathrm{m})$ high resolution $(\approx 1500)$ SWS spectra is also currently under way. Here we focus on the analysis of full range SWS spectra at a resolution of $\lambda / \Delta \lambda \approx 400$ of a set stellar sources that do not show signatures of the presence of dust. The sources were chosen on the basis of their MK classification and cover B to M spectral types. We interpret the observed spectral features in the light of recent stellar model atmospheres (e.g., Decin 2000; Decin et al. 2000) that produce synthetic spectra from which the contribution of molecular bands as a function of stellar parameters can be derived. For stars cooler than M4, the results of these models must be taken with caution since hydrodynamical effects may be significant. We outline a midinfrared classification scheme and correlate the stellar spectral characteristics to the temperature and luminosity of a star of a given spectral type. An additional result from the continuum determinations for the various SWS spectra is the estimates of the stellar angular diameters. We discuss the procedure used to obtain these estimates and the accuracy of the results.

Section 2 describes the observations, data reduction, and continuum determination for the stars selected for this study. A summary is also given of the characteristics of the observed spectra and the spectral classification scheme we derived for these stars. Section 3 contains a quantitative analysis of the molecular bands as a function of MK class. The conclusions are listed in Sect. 4.

\section{Data}

The observations were obtained in the SWS01 observing mode, which covers the entire 2.38-45.2 $\mu \mathrm{m}$ SWS spectral range at spectral resolutions of 1000 to 200 (depending on observation and wavelength). The stars, listed in Table 1, have been selected based on the criteria that they: (i) show no dust features in their mid-ir spectra; (ii) populate a grid of MK spectral types from $\mathrm{B}$ to $\mathrm{M}$; (iii) have a signal to noise ratio high enough to distinguish the atomic and molecular features. The second column in Table 1 lists the MK class for each object, the third column the IRAS LRS class, and the fourth column contains the unique ISO TDT number for the respective observations; this number identifies the observations in the ISO archive. As can be seen, the MK classes are well covered with the exception of the M dwarfs and the cool supergiants. Unfortunately, the M dwarfs were too dim to be observed with SWS. PHOT-S spectra were obtained for the brighter M dwarfs under the STARTYPE ISO experiment. However, these observations have not been included in the present analysis as we wished to preserve consistency of the data base with regard to spectral coverage and resolution. All the cool supergiants initially selected for our sample show strong dust features and have therefore been excluded.

The data have been reduced with the SWS Interactive Analysis (IA3) software (e.g. Salama et al. 1997; Lahuis et al. 1998), using the OLP V10.1 calibration files and algorithms. This version of the software and calibration files includes a correction for memory effects in detector band 2 , which is based on a phenomenological model of the $\mathrm{Si}: \mathrm{Ga}$ detector response (Kester et al. 2001). We flat fielded the Auto-Analysis result, that is, we have aligned the flux profiles of detectors and scans using a fitted polynomial as reference level. We eliminated those points with a deviation from the mean flux value in fixed size bins greater than $3 \sigma$. We also interactively removed segments of the spectra in which a detector exhibited anomalous behavior or where the signal abruptly changed. The final step was to rebin the interactively processed spectra to a common wavelength grid and spectral resolution. A resolution of 400 was selected for the $2.38-12 \mu \mathrm{m}$ spectral range and 250 for wavelengths between 12 and $45.2 \mu \mathrm{m}$. Although some of the observations were taken at higher resolutions, the data were rebinned to preserve consistency in the sample of selected stars. Unfortunately, the flux in the $27.5-45.2 \mu \mathrm{m}$ interval is very low for most of the selected sources and the resulting observations were at a rather low signal-to-noise ratios. Consequently, this wavelength interval has been discarded and the analysis focused on the $2.38-27.5 \mu \mathrm{m}$ wavelength regime.

\subsection{Continuum determination}

An accurate determination of the continuum is required for the analysis of the spectra. However, determining the continuum is not straightforward due to the presence of broad molecular bands in the mid-ir spectra and the peculiar characteristics of the observational data. The SWS spectra are subdivided in wavelength intervals by the manner in which the spectrometer obtained the measurement. These segments, labeled AOT (Astronomical Observation Template) bands, are instrumentally defined by aperture, spectral order, filter band pass and detector type. Also, the accuracy in the flux calibration is different for each band due to the intrinsic characteristics of the instrument, ranging from $2 \%$ at the shortest wavelengths $(2.38$ $4.08 \mu \mathrm{m}$ ), through $6 \%$ between 4.08 and $12 \mu \mathrm{m}$, to $12 \%$ at the longest wavelengths considered. At the AOT band limits the flux calibration is less accurate due to edge effects (worst values are $7 \%$ at the shortest wavelengths, and $15 \%$ at the longest wavelengths). Additionally, observation dependent parameters such as the flux, pointing and dark current measurements, affect each AOT band differently. As a result a spectrum may 

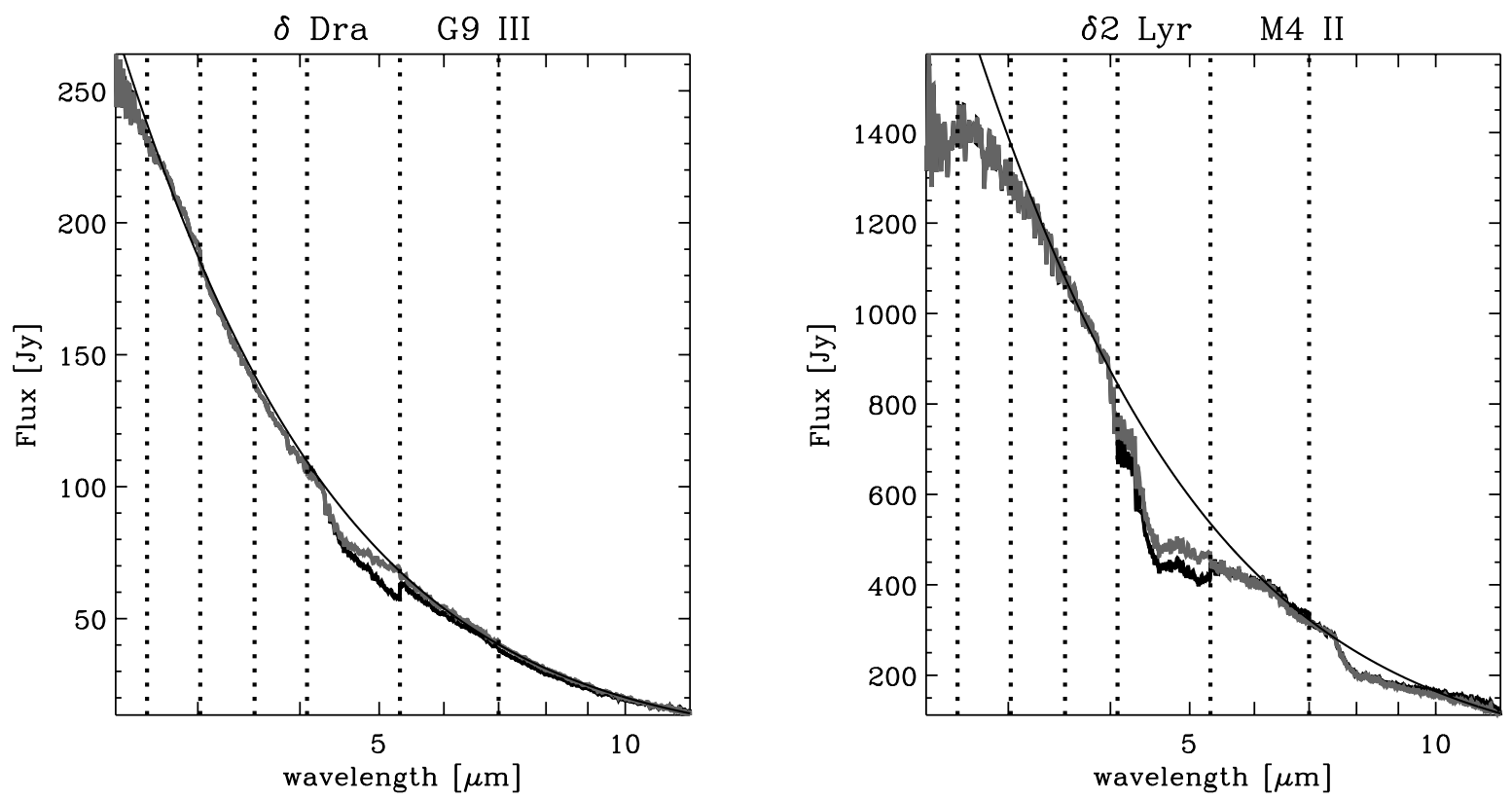

Fig. 1. Correction of AOT band edge discontinuities and the Engelke function fit to the continuum for two representative sources. The observed rebinned spectrum is represented by a thick black line, Engelke's function fit by a thin black line and the rebinned spectrum corrected for AOT band discontinuities is displayed with a grey line. The AOT band limits are indicated by vertical dashed lines.

show discontinuities at the AOT band limits, which adds to the uncertainty in determining the continuum. We fitted the analytical approximation proposed by Engelke (1992) to the $2-60 \mu \mathrm{m}$ infrared continua of the observed spectra, $\phi$. The fit provides a relatively low weight for the band edge discontinuities.

$$
\begin{aligned}
\phi\left(\lambda, T_{\text {eff }}\right)= & \frac{11910 \Omega}{\lambda^{5}\left(\exp \left[19500 / \lambda T_{\text {eff }}\left(1+79450 / \lambda T_{\text {eff }}\right)^{0.182}\right]-1\right)} \\
& \times \mathrm{W} \mu \mathrm{m}^{-1} \mathrm{~cm}^{-2}
\end{aligned}
$$

where wavelength $\lambda$ is in $\mu \mathrm{m}$, effective temperature $T_{\text {eff }}$ is in $\mathrm{K}$, and the solid angle subtended by the star $\Omega$ is in steradian. This function is based on the implicit analytical scaling of a semi-empirical solar atmospheric temperature profile to differing effective temperatures. By fitting this function to a set of standard stars, Engelke (1992) found that the probable error in the estimated flux was $\pm 3 \%$ below $10 \mu \mathrm{m}$ up to $\pm 5 \%$ in the vicinity of $25 \mu \mathrm{m}$, and that the relative flux ratios computed using the continua approximation to a variety of stars agree with the relative photometry of those stars within $\pm 1 \%$ to $\pm 2 \%$. Decin (2001, private communication), compared the continua derived with Engelke's function and continua calculated with the MARCS code for stellar atmospheric models (Gustafsson et al. 1975; Plez et al. 1992), which assume spherical or planeparallel stratification in homogeneous stationary layers, hydrostatic equilibrium and LTE. The comparison showed discrepancies up to $4 \%, 2 \%$ and $7 \%$, for $T_{\text {eff }}$ equal to 10000,6000 and $3500 \mathrm{~K}$, respectively. The amount and sign of these discrepancies were wavelength, temperature and gravity dependent. For example, for a $T_{\text {eff }}$ of $4000 \mathrm{~K}$, and $\log g$ equal to 2, Engelke's function gave flux values which were $5 \%$ lower, at $\approx 2.5 \mu \mathrm{m}$, and $3 \%$ higher, at $\approx 10 \mu \mathrm{m}$, than the model atmospheres, when both distributions were normalized to their flux at $3.9 \mu \mathrm{m}$.

The continuum of each source has been derived by a one parameter fit of Engelke's function to each observed spectrum, leaving the angular diameter as a free parameter. We did not do a two parameter fit because the discontinuities at the AOT band edges introduce too much uncertainty in the fitting procedure. The effective temperature for each star was taken from the literature as representative for its MK class; the adopted values and their references are shown in Table 2. Although different temperature scales have been adopted, they are consistent within their respective errors. Blackwell \& Lynas-Grey (1994) calculated an error of $2 \%$ for their temperature estimates. Ridgway et al. (1980) derived that the internal accuracy of the effective temperatures in their temperature scale was $250 \mathrm{~K}$. Dyck et al. (1996) estimated that the uncertainty for their scale at each spectral type was $95 \mathrm{~K}$. These authors also found that the luminosity class I stars were roughly $400 \mathrm{~K}$ cooler than their luminosity III counterparts at spectral type K4.5. This result has been used to extrapolate the temperature values from class III to class II. The difference between Blackwell \& Lynas-Grey (1994) values and those from Zombeck (1982) is \pm 200 K. Ridgway et al. (1980) concluded that the absolute temperature scale derived from their measurements of stellar diameters was basically the same as that based in the infra-red flux method (Blackwell \& Lynas-Grey 1994). Likewise Dyck et al. (1996) reported an excellent agreement between Michelson interferometry temperatures used in their scale and the lunar occultation temperatures determined by Ridgway et al. (1980).

Engelke's function does not include the contribution to the opacity of the $\mathrm{CO}, \mathrm{SiO}$, and $\mathrm{H}_{2} \mathrm{O}$ absorption bands that are present in the spectra of the cooler stars. Therefore in the fitting procedure we assigned very low weights to those wavelength ranges in which the molecular bands are dominant, specifically $2.38-2.9 \mu \mathrm{m}$ and 4-10 $\mu \mathrm{m}$ (e.g. Decin et al. 1997). Furthermore, since water vapor absorption can lower the continuum in M stars by as much as 5\% (Decin et al. 1997; Decin 2000), we multiplied the M0-M4 type spectra (adopted $T_{\text {eff }}$ 
Table 1. Observed sources.

\begin{tabular}{|c|c|c|c|}
\hline Star & MK Class & LRS class & ISO TDT \# \\
\hline$\alpha \mathrm{Vir}$ & $\mathrm{B} 1 \mathrm{~V}$ & - & 08201001 \\
\hline$\alpha$ Eri & B3 V & 18 & 17902503 \\
\hline$\alpha \mathrm{CMa}$ & $\mathrm{A} 1 \mathrm{~V}$ & 18 & 68901202 \\
\hline$\beta$ Leo & $\mathrm{A} 3 \mathrm{~V}^{\mathrm{b}}$ & - & 04001710 \\
\hline$\beta$ Cas & A5 III & _ & 28501420 \\
\hline$\alpha \mathrm{Aql}$ & A7 V & 18 & 18100805 \\
\hline$\alpha \mathrm{Car}$ & $\mathrm{F} 0 \mathrm{II}^{\mathrm{a}}$ & 18 & 72902207 \\
\hline$\alpha \mathrm{UMi}$ & F7 Ib-II & 18 & 36802830 \\
\hline 44 Dra & F7V & - & 56300507 \\
\hline$\theta$ Per & F7 V & - & 64900206 \\
\hline$\alpha \mathrm{Aqr}$ & $\mathrm{G} 2 \mathrm{Ib}$ & 19 & 17300749 \\
\hline$\beta$ Dra & G2 II & 18 & 08001631 \\
\hline$\beta \mathrm{Hyi}$ & $\mathrm{G} 2 \mathrm{IV}^{\mathrm{d}}$ & 18 & 85000604 \\
\hline$\alpha \mathrm{Cen}$ & $\mathrm{G} 2 \mathrm{~V}$ & - & 60702006 \\
\hline$\delta \mathrm{Pav}$ & G7 IV & - & 29902110 \\
\hline$\eta$ Dra & G8 III & 18 & 08000921 \\
\hline$\delta$ Dra & G9 III & 18 & 20601232 \\
\hline$\alpha \mathrm{UMa}$ & K0 IIIab & 18 & 14300723 \\
\hline$\beta$ Cap & K0 II ${ }^{\mathrm{a}}$ & 18 & 14400108 \\
\hline$\theta$ Cen & K0 III & 19 & 43600940 \\
\hline$\delta$ Eri & K0 IV ${ }^{\mathrm{a}}$ & 18 & 66301815 \\
\hline HR 365 & $\mathrm{~K} 1 \mathrm{~V}^{\mathrm{d}}$ & 18 & 41602312 \\
\hline$\alpha$ Boo & $\mathrm{K} 1.5 \mathrm{III}^{\mathrm{c}}$ & 18 & 45200101 \\
\hline$\xi$ Dra & K2 III & 19 & 31404910 \\
\hline$\alpha$ Ari & K2 III & 18 & 45002411 \\
\hline$\sigma \mathrm{Oph}$ & K3 II & 17 & 10200835 \\
\hline$\gamma$ And & $\mathrm{K} 3 \mathrm{IIb}^{\mathrm{b}}$ & 18 & 43502924 \\
\hline$\lambda \mathrm{Gru}$ & $\mathrm{K} 3 \mathrm{III}^{\mathrm{a}}$ & 17 & 53904837 \\
\hline$\alpha$ Tuc & K3 III & 18 & 86602401 \\
\hline$\beta$ UMi & K4 III & 18 & 18205639 \\
\hline$\gamma$ Phe & $\mathrm{K} 4 / 5 \mathrm{III}^{\mathrm{a}}$ & 16 & 54901434 \\
\hline$\gamma$ Dra & K5 III & 18 & 81100302 \\
\hline$\alpha$ Tau & K5 III & 18 & 63602102 \\
\hline HD 149447 & K5 III ${ }^{\mathrm{a}}$ & 18 & 84700107 \\
\hline$\delta$ Psc & K5 III & - & 39502401 \\
\hline$\beta$ And & M0 III & 18 & 79501002 \\
\hline$\mu \mathrm{UMa}$ & M0 III & - & 16000806 \\
\hline HR 48 & M1 III ${ }^{\mathrm{a}}$ & 19 & 55502138 \\
\hline HR 5301 & M1 III ${ }^{\mathrm{a}}$ & - & 08200810 \\
\hline$\delta \mathrm{Oph}$ & M1 III & 18 & 08201231 \\
\hline$\alpha \mathrm{Cet}$ & M2 III & 18 & 79702803 \\
\hline$\beta$ Peg & M2.5 II-III & 18 & 55100705 \\
\hline$\delta$ Vir & M3 III & 18 & 24201225 \\
\hline$\rho$ Per & M3 III & 18 & 79501105 \\
\hline$\delta^{2} \mathrm{Lyr}$ & M4 II & 18 & 10200126 \\
\hline HR 877 & $\mathrm{M} 4 \mathrm{III}^{\mathrm{d}}$ & 17 & 64900829 \\
\hline$\gamma \mathrm{Cru}$ & M4 III & 18 & 07901307 \\
\hline $57 \mathrm{Peg}$ & $\mathrm{M} 4 \mathrm{~Sv}^{\mathrm{d}}$ & 18 & 37600306 \\
\hline HR 5192 & M4.5 III & 18 & 08101808 \\
\hline TU CVn & M5 III & - & 16001527 \\
\hline$\beta$ Gru & M5 III & - & 53802302 \\
\hline R Lyr & M5 III & - & 53000214 \\
\hline HR 7509 & M5 IIIa & 18 & 74005215 \\
\hline HD 98434 & M6 III ${ }^{\mathrm{a}}$ & 17 & 07901133 \\
\hline$v \mathrm{Pav}$ & M6 III ${ }^{\mathrm{a}}$ & 18 & 12103028 \\
\hline V Nor & M6 III ${ }^{\mathrm{a}}$ & 17 & 45901136 \\
\hline RZ Ari & M6 III & 18 & 46601705 \\
\hline OP Her & M6 Sv & 17 & 77800625 \\
\hline
\end{tabular}

The MK classes are from Jaschek (1978) except for:

\footnotetext{
${ }^{a}$ Michigan Catalogue of two dimensional spectral types for the HD stars.

b Garcia (1989).

c Keenan \& McNeil (1989).

d The Hipparcos Catalogue (ESA 1997).
}

from 3878 to $3595 \mathrm{~K}$ ) by 0.02 and the M5-M6 spectra (adopted $T_{\text {eff }}$ are 3470 and $3380 \mathrm{~K}$, respectively) by 0.05 before performing the fit. Note that, in contrast to the shorter wavelengths, the fitting procedure is more secure at the longer wavelengths, as
Table 2. Adopted effective temperatures for the spectral types.

\begin{tabular}{lll}
\hline \hline MK class & $T_{\text {eff }}(\mathrm{K})$ & Ref. \\
\hline B1 V & 24200 & 1 \\
B3 V & 18800 & 1 \\
A1 V & 10265 & $1^{*}$ \\
A3 V & 8990 & $1^{*}$ \\
A5 III & 8470 & 2 \\
A7 V & 8190 & 1 \\
F0 II & 7350 & 5 \\
F7 Ib-II & 5850 & $1^{*}$ \\
F7 V & 6314 & $1^{*}$ \\
G2 I & 5127 & 2 \\
G2 II & 5100 & 2 \\
G2 IV & 5780 & 1 \\
G2 V & 5780 & 1 \\
G7 IV & 5530 & $1^{*}$ \\
G8 III & 4930 & 3 \\
G9 III & 4860 & $3^{*}$ \\
K0 IIIa & 4790 & 3 \\
K0 II & 4590 & $3^{*}$ \\
K0 III & 4790 & 3 \\
K0 IV & 5015 & $1^{*}$ \\
K1 V & 5010 & $1^{*}$ \\
K1.5 III & 4440 & $4^{*}$ \\
K2 III & 4370 & 4 \\
K3 II & 4230 & 4 \\
K3 III & 4230 & 4 \\
K4 III & 4090 & 4 \\
K4/5 III & 4010 & $4^{*}$ \\
K5 III & 3920 & 4 \\
M0 III & 3878 & $4^{*}$ \\
M1 III & 3835 & 4 \\
M2 III & 3740 & 4 \\
M2.5 II-III & 3700 & $4^{*}$ \\
M3 III & 3675 & 4 \\
M4 II & 3595 & 4 \\
M4 III & 3595 & 4 \\
M4.5 III & 3532 & 4 \\
M5 III & 3470 & 4 \\
M6 III & 3380 & 4 \\
\hline I I & & \\
\hline
\end{tabular}

(1) Zombeck (1982); (2) Blackwell \& Lynas-Gray (1994);

(3) Ridgway et al. (1980); (4) Dyck et al. (1996); (5) Decin (2000).

* Values calculated by interpolation or extrapolation.

there are no significant spectral features over the broad wavelength range between 12 and $29 \mu \mathrm{m}$ and the AOT band discontinuities are relatively small.

Finally the data have been corrected by flat fielding the observed spectra. That is adjusting the data to the continuum derived through the fit, taking care to suppress the AOT band edge jumps while altering the in-band continuum as little as possible. Figure 1 shows the final processed results for two sources, one with moderate and the other with strong molecular 
Table 3. Comparison of the angular diameters $\theta$ derived through Engelke's function fit to the SWS spectra and other values in the literature.

\begin{tabular}{|c|c|c|c|c|c|c|c|}
\hline Star & $\begin{array}{l}\text { Derived } \theta \\
\text { (mas) }\end{array}$ & $\begin{array}{l}\theta \text { other methods } \\
\text { (mas) }\end{array}$ & Ref. & Star & $\begin{array}{l}\text { Derived } \theta \\
\text { (mas) }\end{array}$ & $\begin{array}{l}\theta \text { other methods } \\
\text { (mas) }\end{array}$ & Ref. \\
\hline$\alpha$ Vir & $0.84 \pm 0.03$ & $0.87 \pm 0.04$ & 1 & $\xi$ Dra & $3.20 \pm 0.22$ & $3.025 \pm 0.060$ & 15 \\
\hline$\alpha$ Eri & $1.45 \pm 0.06$ & $1.92 \pm 0.07$ & 1 & & & $3.09 \pm 0.12$ & 18 \\
\hline \multirow[t]{4}{*}{$\alpha \mathrm{CMa}$} & $5.82 \pm 0.37$ & $5.89 \pm 0.16$ & 1 & $\alpha$ Ari & $7.19 \pm 0.52$ & $6.85 \pm 0.07$ & 5 \\
\hline & & 6.20 & 2 & & & $5.9 \pm 0.6$ & 12 \\
\hline & & $5.92 \pm 0.09$ & 3 & & & $8.7 \pm 0.5$ & 14 \\
\hline & & $6.17 \pm 0.27$ & 18 & & & $6.79 \pm 0.23$ & 20 \\
\hline \multirow[t]{3}{*}{$\beta$ Leo } & $1.31 \pm 0.09$ & $1.39 \pm 0.03$ & 4 & & & $7.6 \pm 1.0$ & 22 \\
\hline & & $1.47 \pm 0.06$ & 18 & & & $6.90 \pm 0.074$ & 23 \\
\hline & & $1.33 \pm 0.10$ & 25 & $\sigma \mathrm{Oph}$ & $3.50 \pm 0.27$ & $3.24 \pm 0.035$ & 23 \\
\hline$\beta$ Cas & $1.79 \pm 0.13$ & - & & $\gamma$ And & $8.19 \pm 0.63$ & $7.84 \pm 0.10$ & 5 \\
\hline \multirow[t]{2}{*}{$\alpha$ Aql } & $3.17 \pm 0.23$ & $2.98 \pm 0.14$ & 1 & & & 7.52 & 11 \\
\hline & & $2.92 \pm 0.16$ & 4 & & & $7.0 \pm 0.6$ & 12 \\
\hline \multirow[t]{3}{*}{$\alpha$ Car } & $7.00 \pm 0.41$ & $6.6 \pm 0.8$ & 1 & & & $7.50 \pm 0.36$ & 13 \\
\hline & & $6.81 \pm 0.20$ & 2 & & & $7.72 \pm 0.24$ & 20 \\
\hline & & $7.22 \pm 0.30$ & 18 & $\lambda \mathrm{Gru}$ & $2.82 \pm 0.21$ & $2.71 \pm 0.030$ & 23 \\
\hline$\alpha \mathrm{UMi}$ & $3.18 \pm 0.23$ & $3.0 \pm 0.4$ & 5 & $\alpha$ Tuc & $6.24 \pm 0.46$ & $6.23 \pm 0.25$ & 18 \\
\hline 44 Dra & $1.51 \pm 0.10$ & - & & & & 6.45 & 19 \\
\hline$\theta$ Per & $1.06 \pm 0.07$ & - & & & & $5.99 \pm 0.064$ & 23 \\
\hline \multirow[t]{3}{*}{$\alpha \mathrm{Aqr}$} & $3.05 \pm 0.16$ & 2.972 & 8 & $\beta \mathrm{UMi}$ & $10.14 \pm 0.80$ & $9.7 \pm 0.8$ & 12 \\
\hline & & 2.95 & 24 & & & $9.86 \pm 0.40$ & 18 \\
\hline & & $3.08 \pm 0.03$ & 26 & & & $8.9 \pm 1.1$ & 22 \\
\hline$\beta$ Dra & $3.44 \pm 0.18$ & 3.27 & 6 & & & 10.4 & 24 \\
\hline$\beta$ Нyi & $2.37 \pm 0.11$ & 2.67 & 24 & $\gamma$ Phe & $6.80 \pm 0.54$ & - & \\
\hline \multirow[t]{2}{*}{$\alpha$ Cen } & $8.75 \pm 0.42$ & 8.52 & 7 & $\gamma$ Dra & $10.51 \pm 0.85$ & $10.16 \pm 0.20$ & 3 \\
\hline & & $8.80 \pm 0.34$ & 18 & & & 10.66 & 7 \\
\hline$\delta \mathrm{Pav}$ & $1.85 \pm 0.09$ & - & & & & $9.7 \pm 0.6$ & 12 \\
\hline$\eta$ Dra & $3.65 \pm 0.20$ & 3.44 & 8 & & & $10.13 \pm 0.24$ & 13 \\
\hline \multirow[t]{2}{*}{$\delta$ Dra } & $3.31 \pm 0.21$ & $3.31 \pm 0.13$ & 18 & & & $10.2 \pm 0.2$ & 14 \\
\hline & & $3.8 \pm 0.3$ & 22 & & & $9.997 \pm 0.20$ & 15 \\
\hline \multirow[t]{3}{*}{$\alpha \mathrm{UMa}$} & $6.57 \pm 0.41$ & 6.5 & 6 & & & $10.07 \pm 0.40$ & 18 \\
\hline & & 7.23 & 7 & & & $10.05 \pm 0.29$ & 20 \\
\hline & & 6.64 & 24 & & & $10.17 \pm 0.27$ & 28 \\
\hline \multirow[t]{2}{*}{$\beta$ Cap } & $3.43 \pm 0.22$ & $3.17 \pm 0.12$ & 9 & $\alpha$ Tau & $21.26 \pm 1.72$ & 21.07 & 3 \\
\hline & & $3.18 \pm 0.15$ & 10 & & & $21.205 \pm 0.21$ & 5 \\
\hline \multirow[t]{2}{*}{$\theta$ Cen } & $5.59 \pm 0.35$ & $5.46 \pm 0.058$ & 23 & & & $21.4 \pm 1.1$ & 6 \\
\hline & & 5.36 & 24 & & & $20.21 \pm 0.30$ & 13 \\
\hline$\delta$ Eri & $2.49 \pm 0.15$ & $2.48 \pm 0.026$ & 23 & & & $20.44 \pm 0.11$ & 16 \\
\hline HR 365 & $2.88^{*} \pm 0.18$ & - & & & & $20.77 \pm 0.83$ & 18 \\
\hline \multirow[t]{10}{*}{$\alpha$ Boo } & $20.93 \pm 1.39$ & $19.99 \pm 0.40$ & 2 & & & $20.89 \pm 0.53$ & 20 \\
\hline & & $20.97 \pm 0.20$ & 3 & & & $21.32 \pm 0.58$ & 28 \\
\hline & & 21.5 & 7 & HD 149447 & $4.86 \pm 0.39$ & $4.75 \pm 0.19$ & 18 \\
\hline & & $20.95 \pm 0.20$ & 13 & & & $4.68 \pm 0.053$ & 23 \\
\hline & & $20.43 \pm 0.41$ & 15 & $\delta$ Psc & $3.86 \pm 0.31$ & $4.75 \pm 1.13$ & 10 \\
\hline & & $20.20 \pm 0.08$ & 16 & & & $3.653 \pm 0.07$ & 15 \\
\hline & & $20.80 \pm 0.83$ & 18 & & & $3.91 \pm 0.17$ & 27 \\
\hline & & 21.3 & 24 & & & & \\
\hline & & $21.12 \pm 0.51$ & 28 & & & & \\
\hline & & $19.1 \pm 1.0$ & 29 & & & & \\
\hline
\end{tabular}


Table 3. continued.

\begin{tabular}{|c|c|c|c|c|c|c|c|}
\hline Star & $\begin{array}{l}\text { Derived } \theta \\
\quad(\text { mas })\end{array}$ & $\begin{array}{c}\theta \text { other methods } \\
\text { (mas) }\end{array}$ & Ref. & Star & $\begin{array}{l}\text { Derived } \theta \\
\quad \text { (mas) }\end{array}$ & $\begin{array}{l}\theta \text { other methods } \\
\text { (mas) }\end{array}$ & Ref. \\
\hline \multirow[t]{9}{*}{$\beta$ And } & $13.97 \pm 1.18$ & $13.806 \pm 0.13$ & 5 & $\delta$ Vir & $10.67 \pm 1.00$ & $9.8 \pm 0.6$ & 12 \\
\hline & & $13.5 \pm 0.7$ & 6 & & & $10.16 \pm 0.51$ & 16 \\
\hline & & $12.2 \pm 0.6$ & 12 & $\rho$ Per & $15.21 \pm 1.43$ & $15.53 \pm 0.17$ & 13 \\
\hline & & $14.35 \pm 0.19$ & 13 & $\delta^{2} \mathrm{Lyr}$ & $11.12 \pm 1.10$ & $9.3 \pm 0.5$ & 12 \\
\hline & & $13.9 \pm 0.2$ & 14 & & & $10.73 \pm 0.91$ & 17 \\
\hline & & $13.219 \pm 0.26$ & 15 & HR 877 & $3.78 \pm 0.37$ & - & \\
\hline & & $13.59 \pm 0.55$ & 18 & $\gamma \mathrm{Cru}$ & $26.57 \pm 2.62$ & $26.14 \pm 0.86$ & 28 \\
\hline & & $13.2 \pm 1.7$ & 22 & $57 \mathrm{Peg}$ & $7.68 \pm 0.76$ & - & \\
\hline & & $13.71 \pm 0.37$ & 28 & HR 5192 & $13.85 \pm 1.38$ & - & \\
\hline \multirow[t]{2}{*}{$\mu \mathrm{UMa}$} & $8.64 \pm 0.73$ & $8.28 \pm 0.24$ & 16 & TU CVn & $6.45 \pm 0.65$ & $6.8 \pm 0.6$ & 12 \\
\hline & & $8.32 \pm 0.28$ & 28 & $\beta$ Gru & $28.11 \pm 2.82$ & $27 \pm 3$ & 21 \\
\hline HR 48 & $5.44 \pm 0.48$ & - & & R Lyr & $16.74 \pm 1.68$ & $15.93 \pm 0.37$ & 13 \\
\hline \multirow[t]{2}{*}{ HR 5301} & $4.62 \pm 0.41$ & $3.97 \pm 0.17$ & 10 & & & $13.3 \pm 0.6$ & 29 \\
\hline & & $4.48 \pm 0.23$ & 20 & HR 7509 & $5.30 \pm 0.53$ & - & \\
\hline \multirow[t]{4}{*}{$\delta \mathrm{Oph}$} & $10.48 \pm 0.93$ & $9.3 \pm 0.6$ & 12 & HD 98434 & $6.27 \pm 0.64$ & - & \\
\hline & & $9.918 \pm 0.2$ & 15 & $v$ Pav & $12.97 \pm 1.32$ & - & \\
\hline & & $10.08 \pm 0.48$ & 16 & V Nor & $6.21 \pm 0.63$ & - & \\
\hline & & $10.03 \pm 0.101$ & 23 & RZ Ari & $10.44 \pm 1.06$ & $10.2 \pm 0.2$ & 10 \\
\hline \multirow[t]{7}{*}{$\alpha$ Cet } & $13.18 \pm 1.23$ & $13.23 \pm 0.24$ & 5 & & & $9.1 \pm 0.5$ & 12 \\
\hline & & $11.7 \pm 0.6$ & 12 & & & $9.8 \pm 0.6$ & 12 \\
\hline & & $12.643 \pm 0.25$ & 15 & OP Her & $6.37 \pm 0.65$ & $6.0 \pm 0.6$ & 12 \\
\hline & & $12.08 \pm 0.60$ & 16 & & & & \\
\hline & & $12.52 \pm 0.50$ & 18 & & & & \\
\hline & & $12.59 \pm 0.36$ & 20 & & & & \\
\hline & & $12.66 \pm 0.36$ & 28 & & & & \\
\hline \multirow[t]{8}{*}{$\beta$ Peg } & $16.84 \pm 1.58$ & $17.98 \pm 0.18$ & 5 & & & & \\
\hline & & $16.75 \pm 0.24$ & 13 & & & & \\
\hline & & $18.4 \pm 0.6$ & 14 & & & & \\
\hline & & $16.727 \pm 0.33$ & 15 & & & & \\
\hline & & $16.76 \pm 0.23$ & 16 & & & & \\
\hline & & $16.88 \pm 0.70$ & 18 & & & & \\
\hline & & $16.72 \pm 0.58$ & 20 & & & & \\
\hline & & $16.98 \pm 0.51$ & 28 & & & & \\
\hline
\end{tabular}

(1) Hanbury-Brown et al. (1974); (2) Blackwell et al. (1980); (3) Di Benedetto (1998); (4) Malagnini \& Morossi (1990); (5) Mozurkewich (1991); (6) Manduca et al. (1981); (7) Engelke (1992); (8) Blackwell \& Lynas-Gray (1994); (9) Ridgway et al. (1977); (10) Ridgway et al. (1980); (11) Welch (1994); (12) Dyck et al. (1998); (13) di Benedetto \& Rabbia (1987); (14) Hutter et al. (1989); (15) Blackwell et al. (1991); (16) Perrin et al. (1998); (17) Ridgway et al. (1992); (18) Decin (2000); (19) Basri \& Linsky (1979); (20) Alonso et al. (2000); (21) Bedding et al. (1994); (22) Faucherre et al. (1983); (23) Cohen et al. (1999); (24) Stencel et al. (1980); (25) Smalley \& Dworetsky (1995); (26) Nordgren et al. (1999); (27) Richichi et al. (1992); (28) Cohen et al. (1996); (29) Dyck et al. (1996). *See discussion section.

bands. A thick black line represents the observed spectrum, the fitted Engelke's function is displayed by a thin black line and the corrected spectra is shown by a grey line. The average deviation of the fitted function with respect to the corrected spectra, in those regions without molecular bands, is about $4 \%$.

An interesting cross-check on the results of the continuum fits described above, is that they can be used to derive stellar angular diameters. As Engelke (1992) noted, fitting his empirical function through the absolute spectra of cool stars produces estimates of effective temperatures and angular diameters which correlate well with results obtained by other means. Table 3 displays the values obtained by the present analysis. The errors were calculated by applying (Decin 2000):

$\frac{\sigma_{<\theta>}}{\theta}=\frac{1}{2} \sqrt{\left(\sigma_{\text {cont }}^{2}+\frac{\sigma_{<T_{\text {eff }}>}^{2}}{T_{\text {eff }}^{2}}\right)}$

where $\theta$ is the angular diameter; $\sigma_{<T_{\text {eff }}>}$ is the uncertainty of the temperature derived from the spectral type, which was taken as $1000 \mathrm{~K}, 500 \mathrm{~K}$ and $500 \mathrm{~K}$ for $T_{\text {eff }}$ equal to 10000,6000 and $3500 \mathrm{~K}$, respectively; and $\sigma_{\text {cont }}$ is the error of the continuum, resulting from the uncertainty in the temperature determination plus Engelke's function deviation with respect to 
the continuum derived with MARCS model atmospheres. The $\sigma_{\text {cont }}$ values that we adopted (Decin 2001, private communication) are $8 \%$ for A stars, $6 \%$ for $\mathrm{F}$ stars, $4 \%$ for $\mathrm{G}$ stars, and values from $7 \%$ to $14 \%$, increasing monotonically with spectral type, for $\mathrm{K}$ and $\mathrm{M}$ stars. Angular diameters found in the literature are listed for comparison in the third and seventh columns of Table 3. As can be seen, the agreement is generally good since most of our values are either within the error bars of other determinations, or of the same order. The discrepancies are in general smaller than $15 \%$ and in many cases they are less than $5 \%$. This result supports the accuracy of the absolute SWS calibration. Half of the objects in Table 3 for which a comparison can be made, have angular diameters derived from the Engelke functional fits that are greater than the values obtained by other methods. That fraction increases to two thirds for $M$ stars. This is expected for $M$ stars because Engelke's plane-parallel atmosphere assumption breaks down for these objects. A similar conclusion was reached by Decin (2000), who calculated the stellar parameters through an iterative process in which synthetic spectra computed from stellar model atmospheres were fitted to SWS spectral data. In particular Decin (2000) found that fits to the Engelke function results angular diameters that are systematically too high for G, $\mathrm{K}$ stars and $\mathrm{M}$ giants. Considering all the assumptions that went into fitting the continua and deriving the angular diameters, we conclude that our angular diameters estimates are acceptable and valid also for those sources without other available measurements (except for the problematic case HR 365 that will be discussed below). The results also confirm the validity of the adopted continuum approximation for our sources.

\subsection{Characteristics of the observed SWS stellar spectra}

Figures 2-4 show the $2.38-12 \mu \mathrm{m}$ SWS normalized spectra of our sources in which band edge discontinuities have been corrected, as explained above. This wavelength range is where most infrared spectral features are found. A clear evolution of the near and mid-infrared spectra as a function of spectral type is evident. The general trend is, as expected, that the strength of the molecular bands increases with later spectral types, with the $\mathrm{M}$ stars showing the strongest molecular absorption bands. Hydrogen (Bracket, Pfund and Humphreys series) and helium lines dominate the infrared spectra of B, A and F stars; no molecular bands are observed in these stars. Unfortunately, our normalized spectral resolution is inadequate for a detailed analysis of the behaviour of hydrogen and helium lines as a function of spectral type. The ISO near infrared SWS spectra taken by Vandenbussche et al. $(1999,2002)$ at the highest spectral resolution capability of the instrument, during a dedicated observation campaign after the helium depletion of the satellite, is better suited to this task; a study of the spectra of OB stars included in this program may be found in Lenorzer et al. (2002).

The atomic lines are still visible in $\mathrm{G}$ stars, in which the $\mathrm{CO}$ first overtone (around $2.5 \mu \mathrm{m}$ ) and the $\mathrm{CO}$ fundamental $(\approx 4.6 \mu \mathrm{m})$ absorption bands appear. The $\mathrm{OH}$ band $(\approx 3-3.56 \mu \mathrm{m})$ becomes apparent in $\mathrm{K}$ stars. The $\mathrm{SiO}$ first
Table 4. Adopted wavelength ranges of the molecular bands for the equivalent widths determination.

\begin{tabular}{lc}
\hline \hline Molecular band & $\begin{array}{c}\text { Wavelength range } \\
(\mu \mathrm{m})\end{array}$ \\
\hline CO first overtone & $2.38-2.45$ \\
CO fundamental & $4.30-4.70$ \\
$\mathrm{SiO}$ first overtone & $4.10-4.30$ \\
$\mathrm{SiO}$ fundamental & $7.60-9.00$ \\
$\mathrm{OH}$ band & $3.02-3.4$ \\
$\mathrm{H}_{2} \mathrm{O}$ band & $6.55-6.70$ \\
\hline
\end{tabular}

overtone $(\approx 4 \mu \mathrm{m})$ and $\mathrm{SiO}$ fundamental $(\approx 8 \mu \mathrm{m})$ are first observed in $\mathrm{K} 0$ stars, in agreement with the results by Cohen \& Davies (1995). The broad water band between 6.4 and around $7 \mu \mathrm{m}$ ( $v_{2}$, bending mode) can be seen in $\alpha$ Ari (K2 III). It is also present in $\beta$ And (M0 III) and $\delta$ Oph (M1 III), but only from spectral type M2 it becomes a common feature in our sample. In particular, the water lines between 6.55 and $6.7 \mu$ m produce a deeper absorption that can be used to characterize these late type stars, and that we will refer to as the $\mathrm{H}_{2} \mathrm{O}$ bending mode feature.

The broad scope of our ISO observational program is to provide a classification scheme for all infrared sources observed with the full SWS spectral range, consequently improving on the various infrared spectral classifications, particularly the LRS schemes. As previously noted, our sources belong to the LRS blue classes (characterized by the flux decreasing with wavelength in the 14-22 $\mu \mathrm{m}$ interval), "featureless spectrum", in particular to the classes labeled 16, 17, 18 and 19 (IRAS Science Team 1986). In the spectral classification system of the ISO-SWS spectra developed by Kraemer et al. (2002), our sources are classified as 1.N (ordinary naked stars) or 1.NO (oxygen-rich naked stars). The variety of features that appear in the SWS spectra for these objects shows clearly the need for a more detailed classification scheme. Considering the spectra shown in Figs. 2-4, we propose a subdivision into the following sub-classes: (1) stars with strong $\mathrm{H}$ lines without other features; (2) strong $\mathrm{CO}$ absorption and no $\mathrm{SiO}$; (3) strong $\mathrm{CO}$ and $\mathrm{SiO}$ features; and (4) strong $\mathrm{CO}, \mathrm{SiO}$ features plus the $\mathrm{H}_{2} \mathrm{O}$ bending mode feature.

\section{Molecular bands}

\subsection{Molecular equivalent widths and MK class}

We calculated the equivalent widths of the selected molecular bands that are specified in Table 4 to quantify the main molecular contributions to the stellar spectra. Note that the band widths in the second column of Table 4 are not the actual wavelength extent of the band; these limits were defined to minimize the contribution from other species in each particular wavelength range based on the predictions of the models of $\mathrm{K}$ and $\mathrm{M}$ stars by Decin et al. (1997) and Decin (2000). Also, some band limits were set in order to minimize residual memory effects on the data. As a special case, the $\mathrm{H}_{2} \mathrm{O}$ bands in $\mathrm{M}$ stars may extend over the $2.38-12 \mu \mathrm{m}$ wavelength range considered and beyond. As noted in the previous section, we accounted for this 

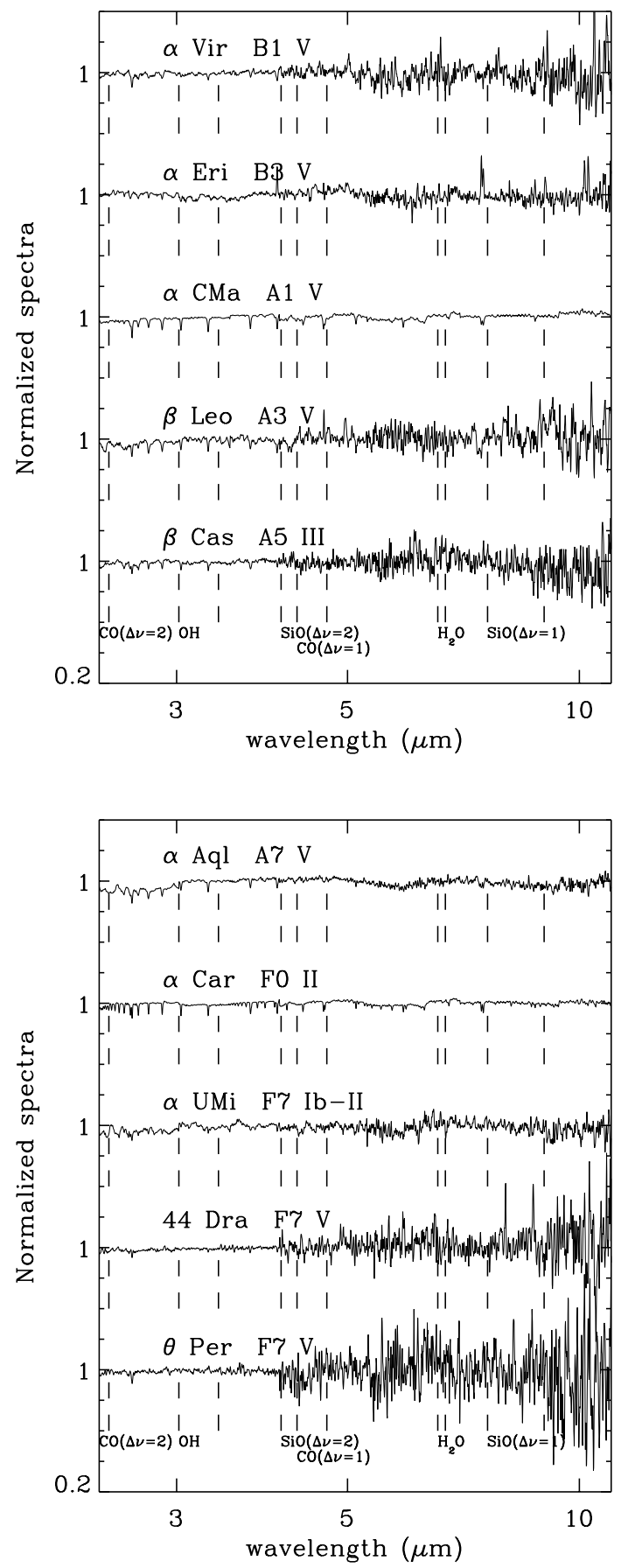
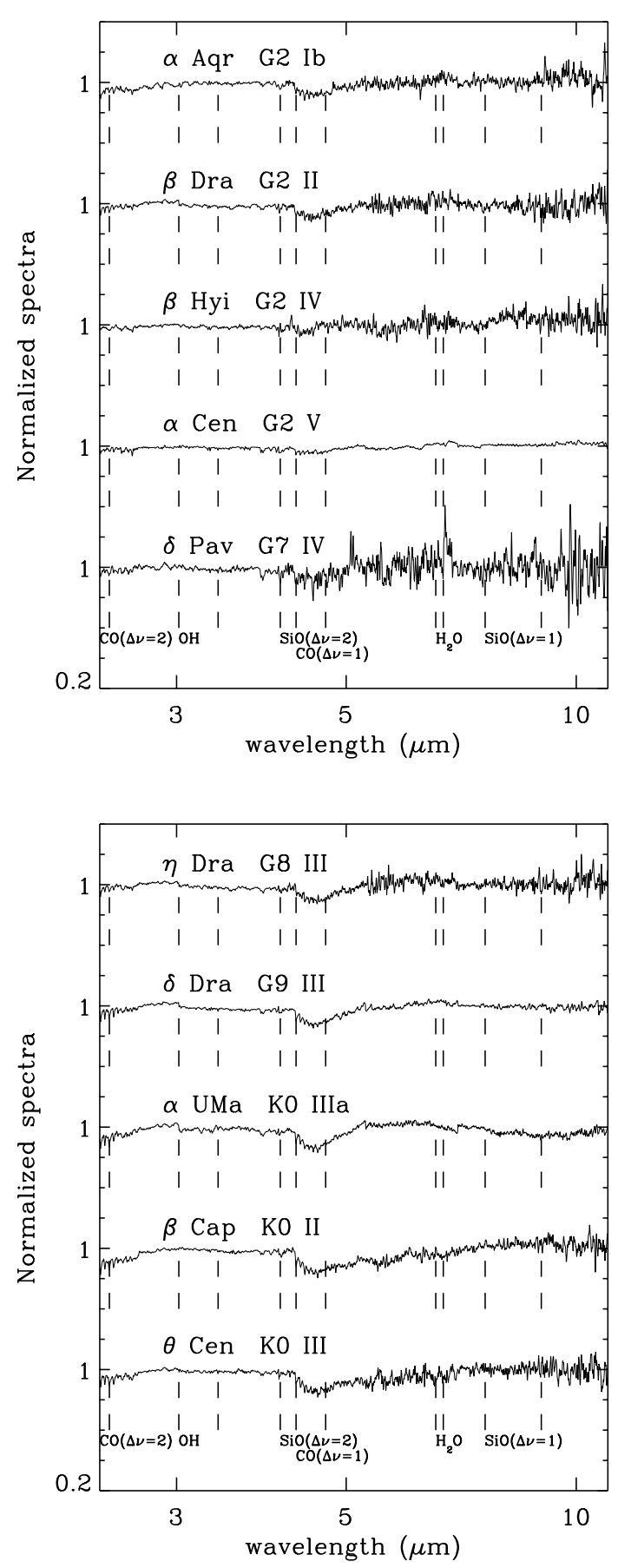

Fig. 2. Spectra normalized to the continuum fit to the Engelke function. The dotted lines indicate the limits of the molecular bands as specified in Table 4 (see text).

quasi-continuous opacity when we derived the continuum through Engelke's function fit, by assuming that the continuum was depressed by $2 \%$ in the M0-M4 stars (adopted $T_{\text {eff }}$ from 3878 to $3595 \mathrm{~K}$ ) and by 5\% in M5-M6 stars (adopted $T_{\text {eff }}$ are 3470 and $3380 \mathrm{~K}$, respectively). Since we flatfield the observational data to the continuum derived through the fit, the assumed water depression is divided out in the normalized spectra. This is a simple approach that does not account for the variation of the water band absorption with wavelength. As this variation cannot be estimated without the use of models, it becomes an additional factor in the uncertainty of the equivalent width estimates. The error bars in the equivalent width calculations include the noise in observed spectra over the wavelength range considered (the standard deviation in the flux), the average uncertainty in the continuum derivation from the fit and a continuum correction factor, where it has been applied. This correction factor is a shift to the normalized spectrum to improve the equivalent width calculation of a particular molecular band, when the deviation from unity of the surrounding continuum was greater than $2 \%$.

As discussed in Sect. 2.1, the slope of Engelke's function can differ a few percent from the slope of the MARCS model 

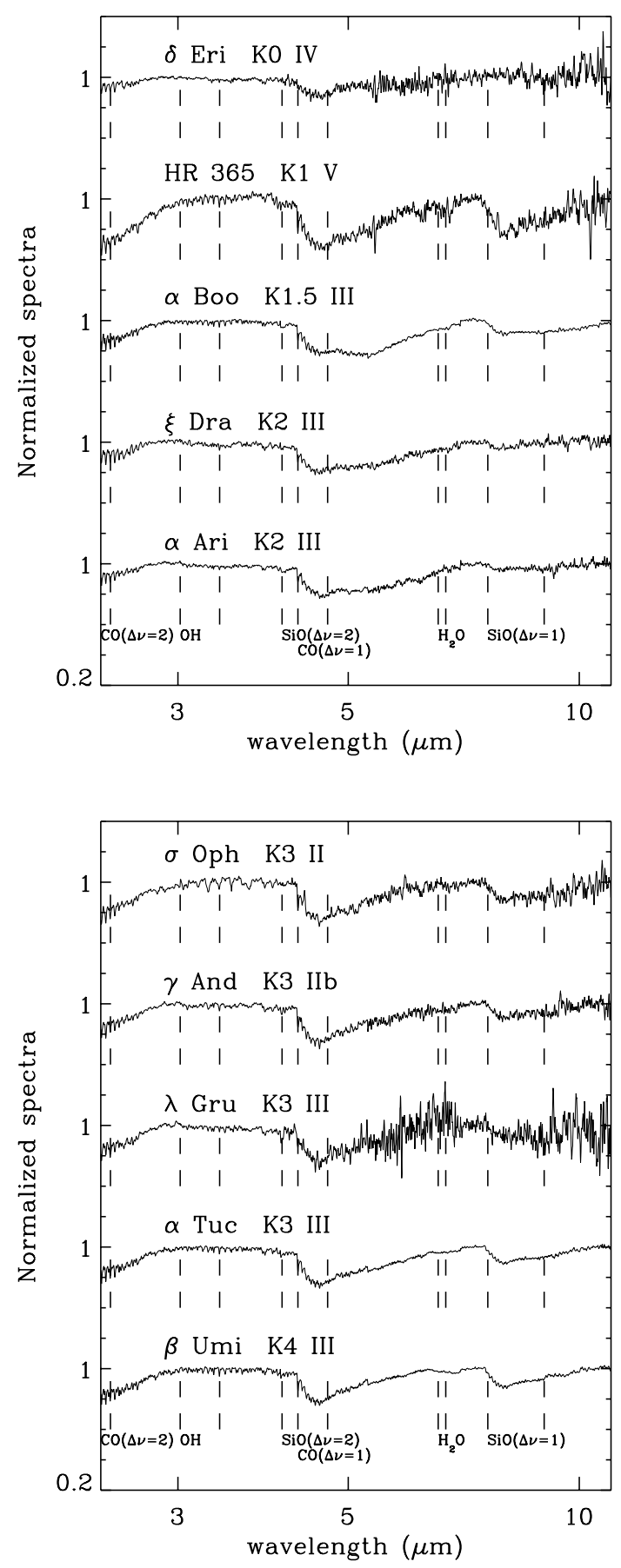

Fig. 3. Continuation of Fig. 2.

synthetic spectrum. As a result the equivalent widths of the $\mathrm{CO}$ first overtone that we have derived may be underestimated by as much as $5 \%$. This systematic deviation has not been included in the error bars. The impact on the equivalent widths of the other bands is negligible because of the flatfielding and normalization of the spectrum that we have applied.

Figure 5 displays the equivalent widths of the $\mathrm{CO}$ first overtone and the $\mathrm{CO}$ fundamental as a function of the stellar spectral type. Both are found to increase with decreasing temperature. The inverse dependence of the band strength with temperature is stronger for $\mathrm{K}$ stars than for $\mathrm{M}$ stars, as can be


seen by the sharp increase in the equivalent widths in $\mathrm{K}$ stars. The subsequent observed flattening of the $\mathrm{CO}$ first overtone band for M stars was previously pointed out by Origlia et al. (1993).

The increase of the $\mathrm{SiO}$ first overtone and $\mathrm{SiO}$ fundamental equivalent widths with decreasing temperatures is illustrated in Fig. 6. Although this trend is present in both bands in $\mathrm{K}$ and $\mathrm{M}$ stars, the dependency of the $\mathrm{SiO}$ first overtone absorption is less pronounced for $\mathrm{K}$ stars, which is consistent with the observations of Rinsland \& Wing (1982). Although there is a general increase of the $\mathrm{SiO}$ fundamental band strength from $\mathrm{K}$ 

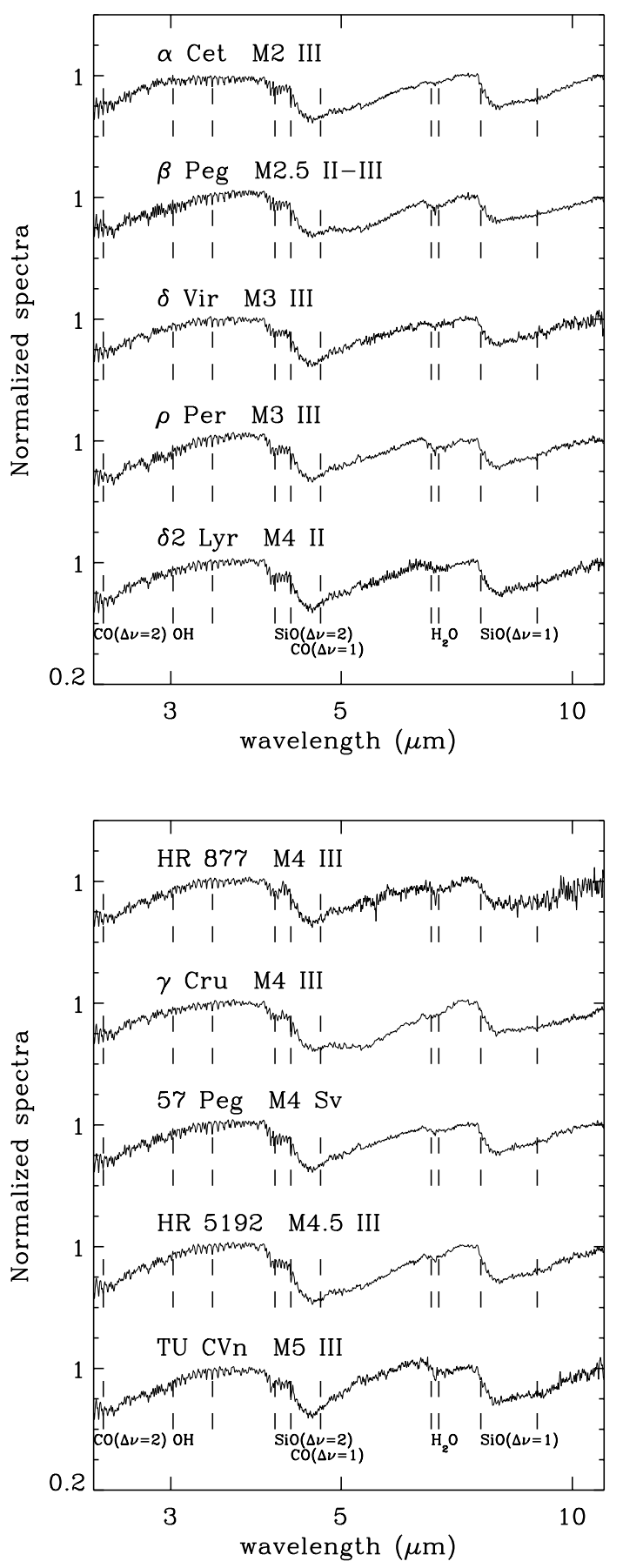

Fig. 4. Continuation of Fig. 3.

to $\mathrm{M}$ stars, a clear trend is not well defined. In particular the equivalent widths in late $\mathrm{K}$ stars and in some M0 to M3 stars are almost equal, in spite of the significant differences in temperature. Two of the five M6 stars and two of the four M5 stars in our sample have $\mathrm{SiO}$ fundamental equivalent widths smaller than M4 stars. That is, the dependence of the SiO fundamental band equivalent width with temperature seems to break down for the cooler stars, where dynamical phenomena, gravity and oxygen abundance are factors of increasing importance.

With regard to the other molecular bands listed in Table 4, some $\mathrm{OH}$ lines are present at spectral types as early as $\mathrm{K} 0$, but it is not until $\mathrm{M}$ stars that a rather significant increase of the


$\mathrm{OH}$ absorption is seen. It is possible to determine the presence of the $\mathrm{H}_{2} \mathrm{O}$ band from our data, but a well defined trend of the equivalent widths as a function of temperature cannot be established.

With reference to the molecular band strengths shown in Figs. 5 and 6, we analyzed what information provided by the moderate resolution SWS $2.38-12 \mu \mathrm{m}$ spectra can be used to infer the spectral type of the star when no dust features are present. We conclude that earlier types (B, A, F) are defined by the relative strength of atomic lines and the absence of molecular signatures (see also Heras et al. 1999; Decin 2000). Spectral type G can be identified by weak CO first overtone and 

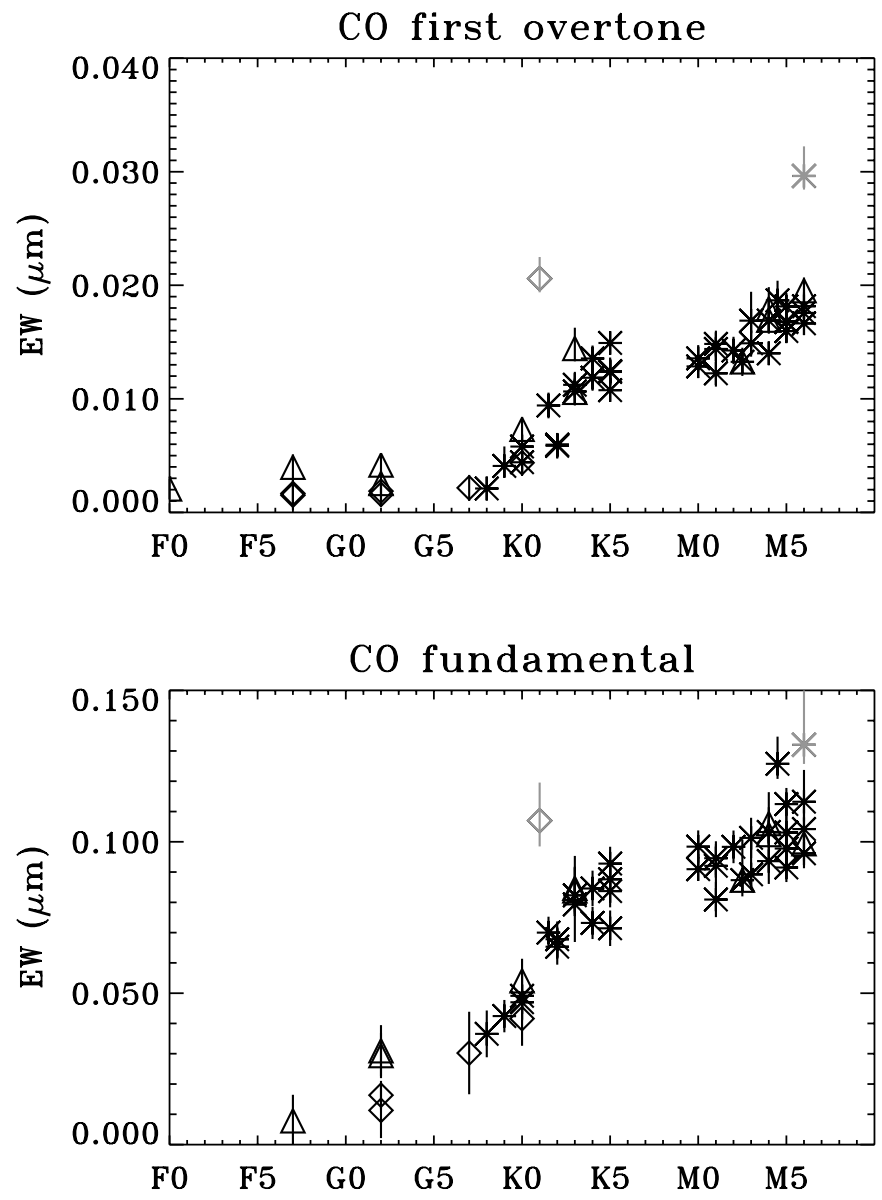

Fig. 5. Equivalent widths of the $\mathrm{CO}$ first overtone and $\mathrm{CO}$ fundamental bands as a function of spectral type. Stars with luminosity classes I and II are represented by triangles, III by asterisks and IV-V by diamonds. HR 365 and V Nor are plotted in grey. The error bars indicate the estimated uncertainty in the standard deviation of the flux and the influence of the errors in determining the equivalent width calculation.

fundamental bands and the absence of $\mathrm{SiO}$ signatures. However our equivalent width data are not enough to determine the subtype, except for the late G8-G9 types. The strengths of the $\mathrm{CO}$ and $\mathrm{SiO}$ bands provide sufficient information to distinguish early K subtypes (K0-K2), "late K-early M" subtypes (K3M3) and late M subtypes (M4-M6). Further subtype separation in the "late $\mathrm{K}$-early M" range can be made by taking the water band at $6 \mu \mathrm{m}$, which appears mainly in M2 and cooler stars. Summarizing, the $\mathrm{CO}, \mathrm{SiO}$ and water spectral bands provide enough information to identify the spectral type and subtype ranges of $\mathrm{K}$ and $\mathrm{M}$ giant stars (since our sample contains basically luminosity classes II and III).

Two objects in our sample, HR 365 (K1 V) and V Nor (M6 III), show a peculiar behaviour. They have been represented by grey symbols in Figs. 5 and 6 , and will be discussed in the following section.

\subsection{Discussion}

The scatter of the equivalent widths with respect to the spectral types in Figs. 5 and 6 is a consequence of several factors. The residuals from inadequate correction of the detector memory effects and AOT band edge discontinuities are a source of uncertainty for the $\mathrm{CO}$ fundamental and the $\mathrm{SiO}$ bands. Also, stellar parameters, such as gravity, metallicity, microturbulent velocity, isotopic ratios and $\mathrm{C}, \mathrm{N}$, and $\mathrm{O}$ abundances, contribute to the dispersion in the equivalent width distributions versus temperature. By computing synthetic spectra with stellar model atmospheres, Decin (2000) analyzed the influence of each one of these parameters on the intensity of the molecular bands in the $2.38-12 \mu \mathrm{m}$ range. Unfortunately, we do not have a large enough sample of sources and the spectral resolution to check the influence of the individual parameters on our observed midinfrared spectra. We can only infer that gravity is a factor that may influence the dependence of the band strength on temperature from the increase in dispersion among supergiants with respect to giants as observed in Figs. 5 and 6. This agrees with the findings by Origlia et al. (1993) for the CO first overtone and from Rinsland \& Wing (1982) for the SiO first overtone lines. Contamination from the circumstellar shell could be a factor that would increase the scatter of the CO fundamental band strength. Tsuji (1986) suggested this to explain the difficulty in matching the observed $\mathrm{CO}$ fundamental fluxes with the predicted ones.

The dependence of the $\mathrm{SiO}$ first overtone absorption as a function of temperature was predicted by stellar model atmospheres (Rinsland \& Wing 1982; Tsuji et al. 1994; Aringer et al. 1997; Decin 2000). With regard to the SiO fundamental, there are few observational studies in the literature due to the difficulties in observing this band from the ground. Cohen et al. (1992) and Cohen \& Davies (1995) reported SiO observations of $\mathrm{K}$ and $\mathrm{M}$ stars taken from the Kuiper Airbone observatory, and with the UKIRT and CGS3 spectrometers, respectively, in which the band strength was seen to increase with later spectral types. Vardya et al. (1986) detected SiO emission in the IRAS LRS spectra of some Mira variables. This discovery is problematic as it was reported before, when Cohen et al. (1992) published the recalibration of the LRS spectra. This recalibration, in part, corrected (by 11\%) the erroneous $\mathrm{SiO}$ emission feature in some stars created by the assumption in the original LRS calibration that $\alpha$ Tau radiated as a $10000 \mathrm{~K}$ blackbody. The present study is the first to sample the $\mathrm{SiO}$ fundamental measurements for normal stars over the whole band wavelength range. We previously pointed out the similarity in the $\mathrm{SiO}$ fundamental equivalent widths in late $\mathrm{K}$ stars, $\mathrm{M} 0$ to M3 and some M6 stars, that is almost independent of the effective temperatures. A similar behaviour was observed in the IRAS-LRS spectra of AGB stars (Sloan \& Price 1998), where the depth of the $\mathrm{SiO}$ fundamental was observed to increase only from $9 \%$ at K5 to $15 \%$ at M6. Likewise, Cohen \& Davis (1995) concluded from CGS3 and UKIRT observations of $\mathrm{K}$ and $\mathrm{M}$ stars, that the $\mathrm{SiO}$ fundamental feature grew essentially linearly for the class III giants, reaching a maximum between K5 III and M0 III, beyond which it remained constant. A stronger dependence on temperature is expected from model atmospheres computations (Aringer et al. 1997). It could be argued that the additional influence of gravity may be the reason for the discrepancy. In the analysis through stellar model atmospheres of $\gamma$ Dra (K5 III) and $\beta$ Peg (M2.5 II-III), Decin (2000) derives the same values for the gravity in these two stars, which 
SiO first overtone

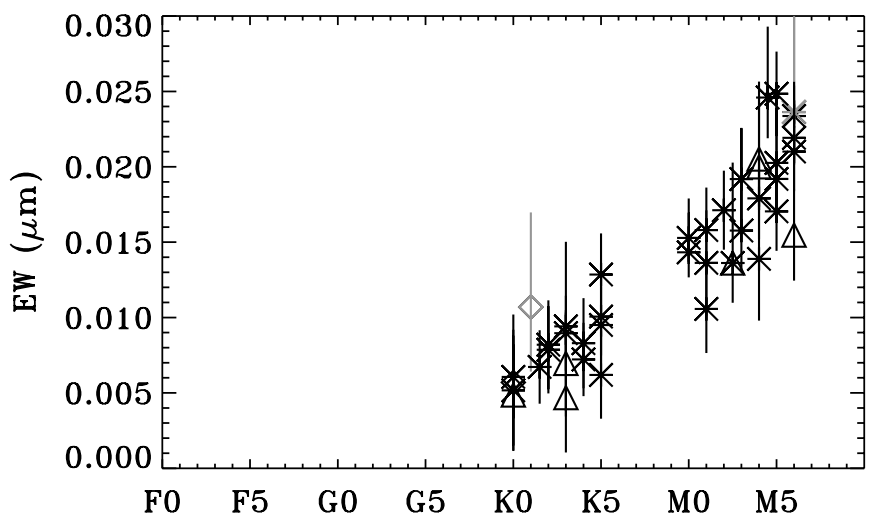

SiO fundamental



Fig. 6. The same as Fig. 5 for the $\mathrm{SiO}$ first overtone and fundamental bands. Stars with luminosity classes I and II are represented by triangles, III by asterisks and IV-V by diamonds.

exhibit almost equal $\mathrm{SiO}$ fundamental equivalent widths despite the difference in their effective temperatures. This example may indicate that gravity is more important than temperature in influencing the $\mathrm{SiO}$ fundamental band strength.

Figure 7 shows that equivalent widths of the various molecular bands are clearly correlated. The correlation of $\mathrm{CO}$ first overtone in $\mathrm{K}$ and $\mathrm{M}$ stars with the $\mathrm{CO}$ fundamental is shown in the top panel, with the $\mathrm{SiO}$ first overtone in the middle and with the SiO fundamental at the bottom. As can be seen in the upper panel, there is a good correlation between both $\mathrm{CO}$ bands over the entire $\mathrm{K}$ through $\mathrm{M}$ sequence. In contrast the $\mathrm{SiO}$ first overtone shows different linear dependences for $\mathrm{K}$ stars and $\mathrm{M}$ stars with respect to the $\mathrm{CO}$ first overtone. The correlation between the $\mathrm{SiO}$ fundamental and the $\mathrm{CO}$ first overtone is the same for K and M stars up to M5, but it seems to be markedly different for M6 stars. We fit a least squares line through the correlations displayed in Fig. 7. The CO fundamental, the $\mathrm{SiO}$ first overtone and the $\mathrm{SiO}$ fundamental band strengths can be estimated from the $\mathrm{CO}$ first overtone band by means of these linear fits. This is of considerable value as the gross features of the infrared spectrum of a star with known spectral type can be estimated from ground based observations of the $\mathrm{CO}$ overtone.
The resulting fits for the indicated spectral types are:

$$
\begin{gathered}
W\left[\mathrm{CO}_{\Delta v=1}\right]=0.033+4.045 W\left[\mathrm{CO}_{\Delta v=2}\right] \\
\text { for } \mathrm{K}-\mathrm{M} \text { stars } \\
W\left[\mathrm{SiO}_{\Delta v=2}\right]=0.005+0.281 W\left[\mathrm{CO}_{\Delta v=2}\right] \\
\text { for } \mathrm{K} \text { stars } \\
W\left[\mathrm{SiO}_{\Delta v=2}\right]=0.001+1.076 W\left[\mathrm{CO}_{\Delta v=2}\right] \\
\text { for } \mathrm{M} \text { stars } \\
W\left[\mathrm{SiO}_{\Delta v=1}\right]=-0.046+15.596 W\left[\mathrm{CO}_{\Delta v=2}\right] \\
\text { for } \mathrm{K}-\mathrm{M} 5 \text { stars. }
\end{gathered}
$$

Because of their anomalous behaviour with respect to their spectral class, we excluded HR 365 (K1 V) and V Nor (M6 III) in deriving these linear dependencies (they are represented by grey points in Figs. 5 and 6). The infrared spectra of both stars are characteristic of later spectral types than the one assigned to them from their optical spectra. The star HR 365 was classified in the GCVS (General Catalogue of Variable Stars, Kholopov et al. 1998) as a semi-regular pulsating star. Its $(B-V)$ index and $V$ magnitude have differing values in the literature. For example, $(B-V)=0.78$, and $V=7.83$ in the Bright Star Catalogue (Hoffleit \& Warren 1991); $V=6.4$ in the WEB Catalog of Radial Velocities (Duflot et al. 1995); $(B-V)=1.915$, and $V=5.91$ in the Tycho Catalogue (ESA 1997); and $(B-V)=$ 2.042, and $V=5.87$ in the Hipparcos Catalogue (ESA 1997). There is also disparity in the optical classification. Appenzeller (1967) firstly classified the star as K1 V. This spectral type is also given in the Hipparcos (ESA 1997), SAO (Ochsenbein 1980) and Bright Star (Hoffleit \& Warren 1991) catalogues. However, HR 365 is classified as M2: III: in the 14th General Catalogue of MK Spectral Classification (Buscombe 1999) and as K4 in the WEB Catalog of Radial Velocities (Duflot et al. 1995). On the other hand, the $(B-V)$ and $(U-B)$ indices listed in the Bright Star Catalogue (Hoffleit \& Warren 1991), are in better agreement with a spectral type G8 V or G2 III, while the $(B-V)$ values given in the Hipparcos and Tycho Catalogues (ESA 1997) indicate a M6 or cooler star. These discrepant data and the disagreement between optical class and infrared spectrum can be mostly explained by the identification of HR 365 as a new double-multiple system in the Hipparcos catalogue (ESA 1997), in which two components separated 0.124". The infrared spectrum is consistent with HR 365 being a double star that is unresolved by the SWS instrument. One star is a G8-K1 dwarf, and provides the optical classification for this object; the other star is of a much later type (M6 III or cooler) and dominates the infrared spectrum. It is this late type star that is responsible for the SWS spectral signature. (The angular diameter of HR 365 given in Table 3 is incorrect since the temperature assumed corresponds to its optical class not that of a late type star.) In this scenario, the different published color indexes, $V$ magnitudes and spectral types are explained by the relative position of the stars with respect to the observer. An interesting point is that the Hipparcos parallax and the range of $V$ values given in the literature match better the binary scenario 
if the blue star is of type G2 III (in agreement with the colour indices in the Bright Star Catalogue, Hoffleit \& Warren 1991), instead of $\mathrm{K} 1 \mathrm{~V}$.

V Nor is classified in the GCVS as a SRb, that is, a semiregular late-type giant with poorly defined periodicity. Since there are no indications of a multiple system, we assume that the variation is intrinsic to the star. Based on the equivalent widths of the $\mathrm{CO}$ first overtone, the $\mathrm{CO}$ fundamental and the $\mathrm{H}_{2} \mathrm{O}$ bending mode band, we infer that the spectral type of this star is later than M6. A plausible explanation is that V Nor is in a more advanced stage of evolution than the other giants in our study, its atmosphere being more affected by phenomena related to pulsation, and showing as a result a slightly different infrared spectrum. On the other hand, Kraemer et al. (2002) classify V Nor as having a thin circumstellar dust shell, which would distort the spectral analysis of this object.

\section{Conclusions}

We analyzed the ISO-SWS low resolution spectra of stars with spectral types B to $\mathrm{M}$, which show no indication of dust features. The continua were defined by fitting Engelke's function to the SWS spectra. These fits lead to acceptable values for the stellar angular diameters. Our work is an additional step in extending and refining infrared spectral classification methodologies based on SWS full range spectra. In this paper we propose a subdivision of the 1.N (ordinary naked stars) and 1.NO (oxygen-rich naked stars) subgroups defined in Kraemer et al. (2002) in the following sub-classes: (1) stars with strong $\mathrm{H}$ lines without no other features; (2) strong $\mathrm{CO}$ absorption and no $\mathrm{SiO}$; (3) strong $\mathrm{CO}$ and $\mathrm{SiO}$ features; and (4) strong $\mathrm{CO}$, $\mathrm{SiO}$ features plus the $\mathrm{H}_{2} \mathrm{O}$ bending mode feature.

The analysis of the equivalent widths of the principal molecular bands in the cooler stars (CO first overtone, $\mathrm{CO}$ fundamental, $\mathrm{SiO}$ first overtone, $\mathrm{SiO}$ fundamental, and $\mathrm{H}_{2} \mathrm{O}$ bending mode) as a function of MK class, leads to the following conclusions:

1. The $2.38-27.5 \mu \mathrm{m}$ moderate resolution $(\approx 400)$ spectra analyzed in this study provide enough information to distinguish between hot (B, A, F) and cool stars. In cool giants, it is possible to determine the spectral type $\mathrm{G}$, and the spectral type ranges $\mathrm{K} 0-\mathrm{K} 2, \mathrm{~K} 3-\mathrm{M} 1, \mathrm{M} 2-\mathrm{M} 3$ and M4-M6.

2. Good correlations were found between the equivalent widths of the molecular bands in $\mathrm{K}$ and $\mathrm{M}$ stars. Empirical linear functions were derived from which the $\mathrm{CO}$ fundamental, the $\mathrm{SiO}$ first overtone and the $\mathrm{SiO}$ fundamental band depths can be estimated from the $\mathrm{CO}$ first overtone. This implies that it is possible to extrapolate the mid-infrared spectrum from $\mathrm{CO}$ first overtone ground observations, if the optical classification of the star is known.

3. We have identified two peculiar objects whose mid-infrared spectrum does not correspond to their optical classification, HR 365 and V Nor. HR 365 has a late M companion, which dominates the observed spectrum. V Nor is a late type giant which may have a circumstellar dust shell.

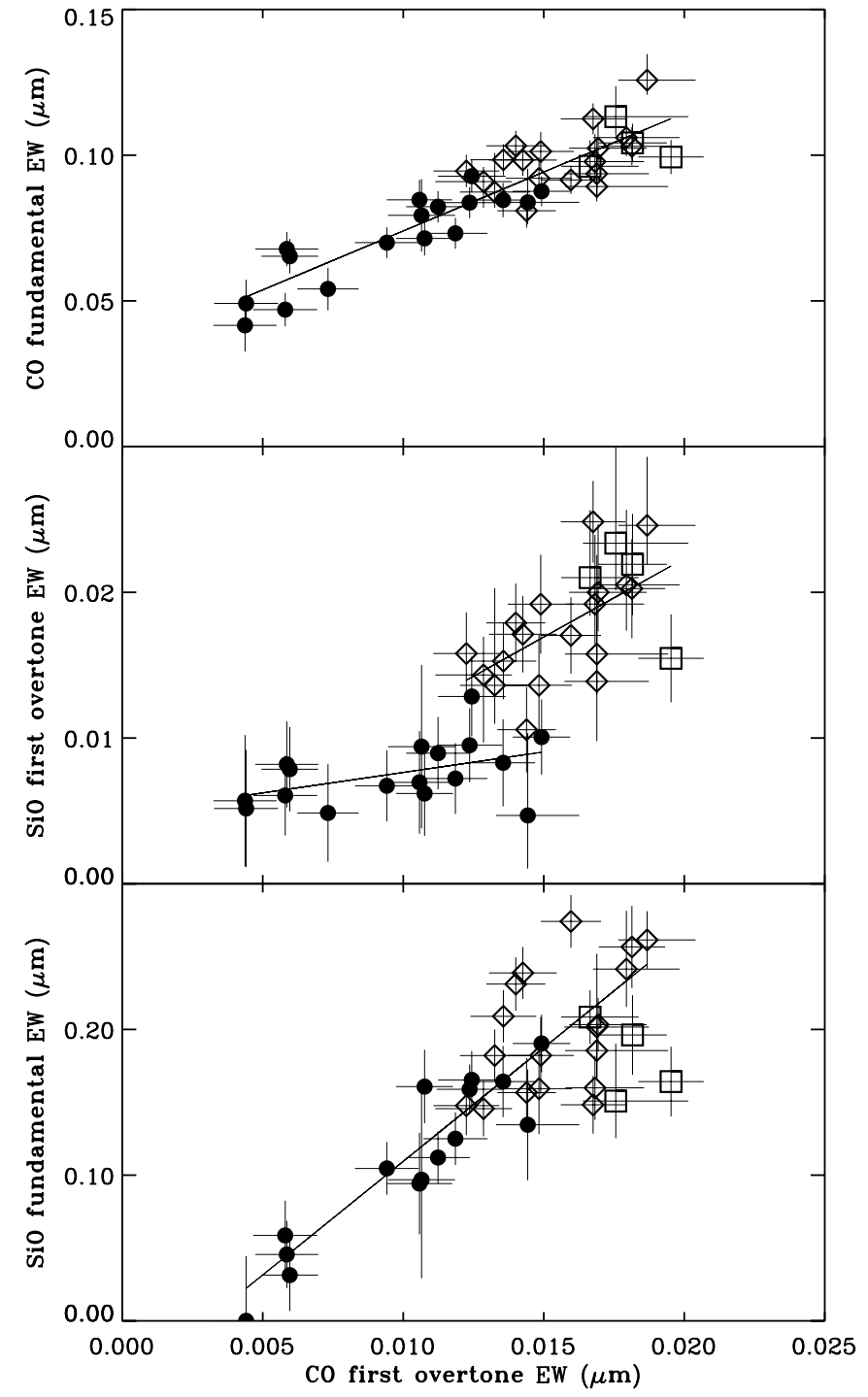

Fig. 7. Correlation between the equivalent widths of various molecular bands with those for the $\mathrm{CO}$ first overtone. $\mathrm{K}$ stars are represented by full circles, M0 to M5 stars by diamonds and M6 stars by squares. The lines represent the best linear fit to the data.

Acknowledgements. IA3 is a joint development of the SWS consortium. Contributing institutes are SRON, MPE, KUL and the ESA Astrophysics Division. This research has made use of NASA's Astrophysics Data System Abstract Service and catalogues available at CDS.

\section{References}

Alonso, A., Salaris, M., Arribas, S., et al. 2000, A\&A, 355, 1060 Andrillat, Y., Jaschek, C., \& Jaschek, M. 1995, A\&AS, 112, 475 Appenzeller, I. 1967, PASP, 79, 102

Aringer, B., Jorgensen, U. G., \& Langhoff, S. R. 1997, A\&A, 323, 202 Aringer, B., Höfner, S., Wiedemann, G., et al. 1999, A\&A, 342, 799

Basri, G. S., \& Linsky, J. L. 1979, ApJ, 234, 1023

Bedding, T. R., Robertson, J. G., \& Marson, R. G. 1994, A\&A, 290, 340

Blackwell, D. E., Petford, A. D., \& Shallis, M. J. 1980, A\&A, 82, 249

Blackwell, D. E., Lynas-Gray, A. E., \& Petford, A. D. 1991, A\&A, 245,567 
Blackwell, D. E., \& Lynas-Gray, A. E. 1994, A\&A, 282, 899

Buscombe, W. 1999, 14th General Catalogue of MK Spectral Classification, Northwestern Univ., Evanston, Illinois ISBN 0-939160-12-9

Cohen, M., Walker, R. G., \& Witteborn, F. C. 1992, AJ, 104, 2030

Cohen, M., Witteborn, F. C., Carbon, D. F., et al. 1992, AJ, 104, 2045

Cohen, M., \& Davies, J. K. 1995, MNRAS, 276, 715

Cohen, M., Witteborn, F. C., Carbon, D. F., et al. 1996, AJ, 112, 2274

Cohen, M., Walker, R. G., Carter, B., et al. 1999, AJ, 117, 1864

Decin, L., Cohen, M., Eriksson, K., et al. 1997, Comparison between ISO-SWS observations and synthetic spectra of $\mathrm{K}$ giants and $\mathrm{M}$ giants $\alpha$ Boo and $\beta$ Peg, in The first ISO workshop on Analytical Spectroscopy, ed. A. M. Heras, K. Leech, N. R. Trams, \& M. Perry, ESA SP-419, 185

Decin, L. 2000, Synthetic spectra of cool stars observed with the Short-Wavelength Spectrometer: improving the models and the calibration of the instrument, Ph.D. Thesis, Katholieke Universiteit Leuven

Decin, L., Waelkens, C., Eriksson, K., et al. 2000, A\&A, 364, 137

Di Benedetto, G. P., \& Rabbia, Y. 1987, A\&A, 188, 114

Di Benedetto, G. P. 1998, A\&A, 339, 858

Duflot, M., Figon, P., \& Meyssonnier, N. 1995, A\&AS, 114, 269

Dyck, H. M., Benson, J. A., van Belle, G. T., et al. 1996, AJ, 111, 1705

Dyck, H. M., van Belle, G. T., \& Thompson, R. R. 1998, AJ, 116, 981

Engelke, C. W. 1992, AJ, 104, 1248

ESA 1997, The Hipparcos and Tycho Catalogues, ESA SP-1200

Faucherre, M., Bonneau, D., Koechlin, L., et al. 1983, A\&A, 120, 263

Garcia, B. 1989, A List of MK Standard Stars, Bull. Inform. CDS 36, 27

Gustafsson, B., Bell, R. A., Eriksson, K., \& Nordlund, A. 1975, A\&A, 42,407

Hanbury Brown, R., Davis, J., \& Allen, L. R. 1974, MNRAS, 167, 121

Heras, A. M., Shipman, R. F., Price, S. D., et al. 1997, Ap\&SS, 255, 251

Heras, A. M., Shipman, R. F., Price, S. D., et al. 1999, SWS spectral classification of ordinary stars, in The Universe as Seen by ISO, ed. P. Cox, \& M. F. Kessler, ESA SP-427, 329

Hoffleit, D., \& Warren, Jr W. H. 1991, The Bright Star Catalogue, 5th Revised Ed., Astronomical Data Center, NSSDC/ADC

Hutter, D. J., Johnston, K. J., Mozurkewich, D., et al. 1989, ApJ, 340, 1103

IRAS Science team 1986, A\&AS, 65, 607

Jaschek, M. 1978, Catalogue of selected spectral types in the MK system, Bull. Inform. CDS 15, 121

Keenan, P. C., \& McNeil, R. C. 1989, ApJS, 71, 245

Kester, D., Fouks, B., \& Lahuis, F. 2001, SWS Transient Effects: Models and Corrections, in The calibration legacy of the ISO Mission, ed. L. Metcalfe, \& M. F. Kessler, ESA SP-481, in press

Kholopov, P. N., Samus, N. N., Frolov, M. S., et al. 1998, Combined General Catalogue of Variable Stars, 4.1
Kraemer, K. E., Sloan, G. C., Price, S. D., \& Walker, H. J. 2002, ApJS, 140,389

Lahuis, F., Wieprecht, E., Bauer, O. H., et al. 1998, ASP Conf. Ser. 145, 224

Lenorzer, A., Vandenbussche, B., Morris, P., et al. 2002, A\&A, 384, 473

Malagnini, M. L., \& Morossi, C. 1990, A\&AS, 85, 1015

Manduca, A., Bell, R. A., \& Gustafsson, B. 1981, ApJ, 243, 883

Meyer, M. R., Edwards, S., Hinkle, K. H., et al. 1998, ApJ, 508, 397

Morris, P. W., Eenens, P. R. J., Hanson, M. M., et al. 1996, ApJ, 470, 597

Mozurkewich, D., Johnston, K. J., Simon, R. S., et al. 1991, AJ, 101, 2207

Nordgren, T. E., Germain, M. E., Benson, J. A., et al. 1999, AJ, 118, 3032

Ochsenbein, F. 1980, SAO and Supplementary Data, Bull. Inf. CDS, 19,74

Origlia, L., Moorwood, A. F. M., \& Oliva, E. 1993, A\&A, 280, 536

Perrin, G., Coude Du Foresto, V., Ridgway, S. T., et al. 1998, A\&A, 331,619

Plez, B., Brett, J. M., \& Nordlund, A. 1992, A\&A, 256, 551

Richichi, A., di Giacomo, A., Lisi, F., \& Calamai, G. 1992, A\&A, 265, 535

Ridgway, S. T., Wells, D. C., \& Joyce, R. R. 1977, AJ, 82, 414

Ridgway, S. T., Joyce, R. R., White, N. M., et al. 1980, ApJ, 235, 126

Ridgway, S. T., Benson, J. A., Dyck, H. M., et al. 1992, AJ, 104, 2224

Rinsland, C. P., \& Wing, R. F. 1982, ApJ, 262, 201

Salama, A., Feuchtgruber, H., Heras, A. M., et al. 1997, Topics in SWS Data Analysis and Calibration. in The first ISO workshop on Analytical Spectroscopy, ed. A. M. Heras, K. Leech, N. R. Trams, \& M. Perry, ESA SP-419, 17

Sloan, G. C., \& Price, S. D. 1998, ApJS, 119, 141

Smalley, B., \& Dworetsky, M. M. 1995, A\&A, 293, 446

Smith, V. V., \& Lambert, D. L. 1985, ApJ, 294, 326

Stencel, R. E., Linsky, J. L., Mullan, D. J., et al. 1980, ApJS, 44, 383

Tsuji, T. 1986, ArA\&A, 24, 89

Tsuji, T., Ohnaka, K., Hinkle, K. H., et al. 1994, A\&A, 289, 469

Vandenbussche, B., Waters, L. B. F. M., Heras, A. M., et al. 1999, The SWS-Post-Helium programme extending the MK classification to the near infrared, in The Universe as Seen by ISO, ed. P. Cox, \& M. F. Kessler, ESA SP-427, 413

Vandenbussche, B., Beintema, D., de Graauw, T., et al. 2002, A\&A, 390, 1033

Vardya, M. S., de Jong, T., \& Willems, F. J. 1986, ApJ, 304, L29

Wallace, L., \& Hinkle, K. 1996, ApJS, 107, 312

Welch, D. L. 1994, AJ, 108, 1421

Zombeck, M. V. 1982, Handbook of space astronomy and astrophysics (Cambridge University Press, Cambridge) 